Applied and

Computational

Mathematics

Division

Computing and Applied Mathematics Laboratory

\title{
Portable Vectorized Software for Bessel Function Evaluation
}

Ronald F. Boisvert and Bonita V. Saunders

June 1991 



\section{Portable Vectorized Software for Bessel Function Evaluation}

\section{Ronald F. Boisvert Bonita V. Saunders}

U.S. DEPARTMENT OF COMMERCE National Institute of Standards and Technology

Computing and Appiled Mathematics Laboratory

Applied and Computational Mathematics Division

Gaithersburc, MD 20899

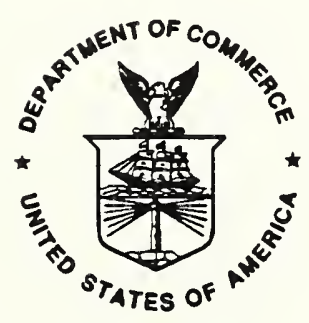

U.S. DEPARTMENT OF COMMERCE Robert A. Mosbacher, Secretary MATONAL INSTIUTIE OF STANDARDS NND TECHNOLOQY

John W. Lyons, Director 



\title{
Portable Vectorized Software for Bessel Function Evaluation
}

\author{
Ronald F. Boisvert and Bonita V. Saunders* \\ Computing and Applied Mathematics Laboratory \\ National Institute of Standards and Technology \\ Gaithersburg, MD 20899 \\ June 19, 1991
}

\begin{abstract}
A suite of computer programs for the evaluation of Bessel functions and modified Bessel functions of orders zero and one for a vector of real arguments is described. Distinguishing characteristics of these programs are that (a) they are portable across a wide range of machines, and (b) they are vectorized in the case when multiple function evaluations are to be performed. The performance of the new programs are compared with software from the FNLIB collection of Fullerton on which the new software is based.
\end{abstract}

Keywords: Bessel function, hyperbolic Bessel function, mathematical software, modified Bessel function, order zero and one, portable software, special function, vectorized software.

\section{Introduction}

Bessel functions of real argument and integer order are among the most commonly occurring special functions of applied mathematics, and most software libraries contain routines for their evaluation. One of the most successful collections of routines for evaluating these and other special functions is the FNLIB package developed by Wayne Fullerton at the Los Alamos National Laboratories in the late 1970s [9]. One of the most important features of FNLIB is its portability. Parameterized by the PORT machine constants [8], FNLIB codes are regularly used on machines from IBM PCs to Cray Y-MPs. Versions of these codes have found their way into several well-known libraries such as the IMSL SFUN/LIBRARY [10], and the SLATEC Common Math Library [2]. They are also available from netlib [6].

\footnotetext{
•Electronic mail: boisvert@cam.nist.gov and saunders@cam.nist.gov, respectively
} 
More recently, increased attention has been paid to the development of algorithms and software which take advantage of vector processors. On such machines, for example, special versions of many standard Fortran math functions are available, so that the compiler can vectorize loops such as the following.

$$
\begin{aligned}
& \text { DO } 10 I=1, M \\
& Y(I)=\operatorname{EXP}(X(I)) \\
& 10 \text { CONTINUE }
\end{aligned}
$$

The ability to vectorize such loops is crucial in many applications.

In this paper we describe a set of Fortran-callable subprograms which extend this functionality to the Bessel functions $I_{0}, I_{1}, J_{0}, J_{1}, K_{0}, K_{1}, Y_{0}$, and $Y_{1}$. This has been done by producing modified versions of the FNLIB routines BESI0, BESI1, BESJ0, BESJ1, BESK0, BESK1, BESY0 and BESY1, as well as their double precision versions. The new routines maintain the portability of FNLIB with the advantage of being vectorizable in cases when multiple function evaluations are required. In Section 2 we review the basic design of the FNLIB routines. In Section 3 we discuss various issues involved in the vectorization of these algorithms. This is followed by a short description of the user interface of our implementation in Section 4. Finally, in Section 5 we describe the testing of the new software, and evaluate its performance on various scalar and vector processors.

\section{Design of FNLIB}

FNLIB is a substantial collection of software, including more than 200 Fortran subprograms. Double precision versions are available for most codes. Primary design criteria for the development of the package were portability and maintainability. In some cases, other criteria such as speed and accuracy were relaxed slightly in order to maximize the primary criteria [9]. For example, FNLIB routines are rarely accurate to the last bit, although they are almost always accurate to within a factor of 10 times the machine precision. This is certainly sufficient for the vast majority of applications. The fact that these routines have remained popular for more than 10 years, and have been trivially ported to many machines which did not exist when they were initially developed attests to the success of the basic design.

FNLIB is based upon approximation by truncated Chebyshev series expansions [7]. Such approximations have many well-known properties: they are widely applicable, they are nearly best in the minimax sense, the error is easy to estimate, they provide variable accuracy approximations, and they are more stable to evaluate than conventional polynomials. Because of these properties, such approximations have been the basis for many algorithms for the evaluation of special functions.

The basic formula for the construction of the routines in FNLIB, which we paraphrase from [9], follows.

\section{Recipe for Constructing FNLIB Routines}

1. Store Chebyshev coefficients in DATA statements.

Coefficients of all required Chebyshev expansions accurate to 16 digits are stored in sin- 
gle precision routines. 31 digits in double precision routines. This range ensures sufficient accuracy for most computers.

2. Initialize.

This is done only on the first call of each routine.

(a) Calculate legal argument bounds.

Mlegal argument regions are those where the function is undefined, the result would overflow or underflow, or range reduction cannot be performed accurately enough. The PORT machine constants are used to determine bounds applicable to the current machine.

(b) Determine correct number of expansion coefficients.

The error committed in truncating a Chebyshev series is bounded by the sum of the absolute values of the discarded coefficients. One can use this to estimate the error committed by using fewer and fewer terms. In this way the length of the series can be selected to match the precision of the machine.

3. Check input argument for validity.

The function argument is compared against bounds computed during initialization. Both fatal errors and warnings are provided. Warnings are issued for underflowed results, for example. while fatal errors are issued in cases where no result can be returned, such as where the result overfows. Errors are issued using the PORT error handler [8].

4. Compute the approximation.

The basic computation is the evaluation of a truncated Chebyshev series. It is not reasonable for a single series to be accurate over the whole argument range. Thus, the argument range is broken up into several subintervals (usually three or four), each with its own series. Once the appropriate subinterval has been determined, the computation proceeds as follows.

(a) Preprocess argument.

The argument is mapped to the interval $[-1,1]$.

(b) Evaluate Chebyshev series.

This is done using a three-term linear recurrence due to Clenshaw [3].

(c) Postprocess result.

The series value is finally corrected for form or interval, if necessary.

\section{Vectorization for Multiple Arguments}

In this section we describe how routines with the above design may be extended for the case of evaluation at multiple arguments. Three opportunities for improving performance exist: (1) reduction of subprogram call overhead, (2) vectorization of pre- and postprocessing phases, and (3) vectorization of Chebyshev series evaluation. The emphasis on this paper is on (2) and (3).

The vectorization of function evaluation routines for multiple arguments seems quite simple at first - one simply applies the scalar algorithm to the vector of arguments. Unfortunately, this fails because the range of possible input arguments is divided into several 
subintervals, each of which is handled differently. Since we cannot assume that the input arguments have been sorted in any way, the core of the algorithm proceeds as follows.

For each argument range:

Gather arguments from this range into a temporary vector.

Compute the approximation for these arguments in vector mode.

Scatter results back into resultant vector.

Clearly, the vector lengths will depend upon the distribution of input arguments, and, in general, will be less than the total number of arguments.

To illustrate this transformation we consider the following linestatement from the FNLIB routine $\mathrm{BESIO}^{1}$.

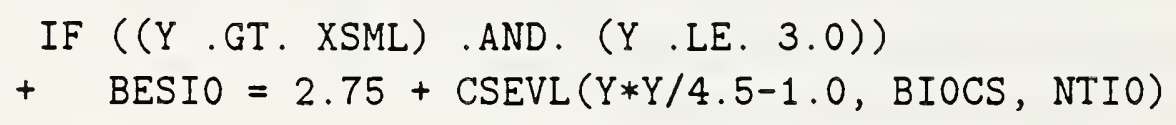

This line illustrates how one particular argument range is handled. The approximation in this case is simply 2.75 plus the Chebyshev series sum returned by the utility CSEVL. BIOCS is an array of Chebyshev series coefficients and NTIO is the number of coefficients. The corresponding vector code is more complex.

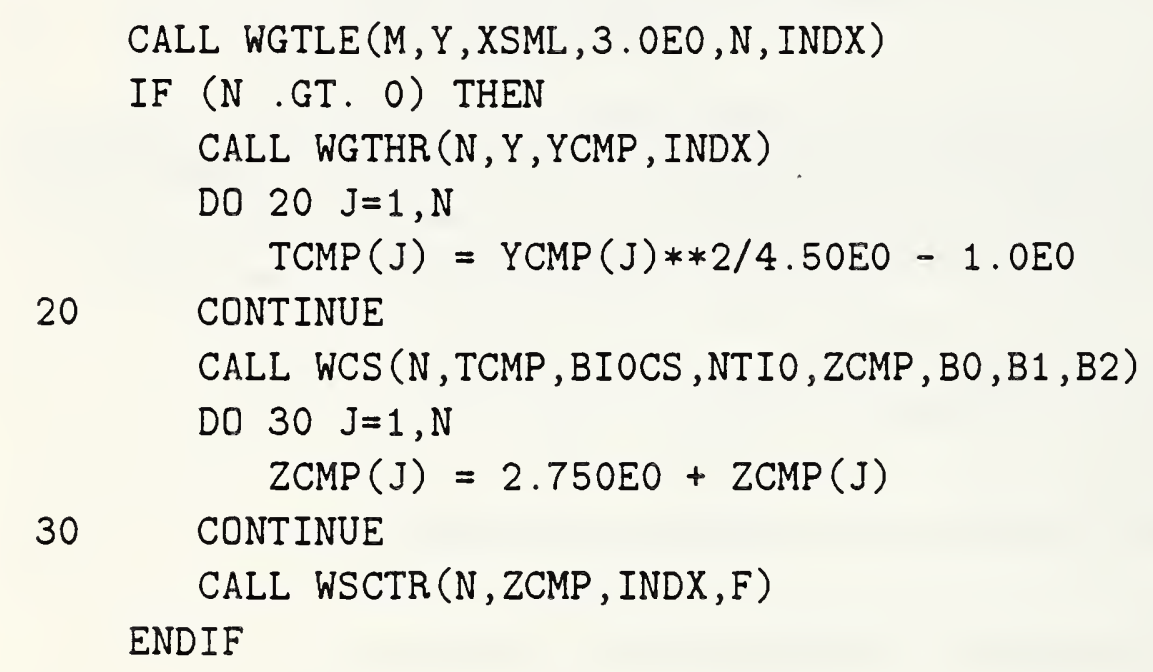

Here there are $M$ arguments in the array $Y$ for which the function is to be evaluated with result returned in the array $F$. WGTLE returns an array INDX of the $N$ indices of elements of the vector $Y$ that are between XSML and 3. These are gathered by the routine WGTHR into YCMP. The 20 loop preprocesses the argument array. WCS evaluates the same Chebyshev series as CSEVL, except for a vector of $\mathrm{N}$ arguments stored in the array TCMP. BO, B1, and B2 are work arrays of length $\mathrm{M}$. The 30 loop postprocesses the result of the series evaluation, and then WSCTR puts the result into the appropriate positions of $F$. The preprocessing and postprocessing phases are trivially vectorizable, while the routines WGTLE, WGTHR, and WSCTR represent utility operations which are also vectorizable.

\footnotetext{
${ }^{1}$ This statement has been modified slightly from the original code to simplify the presentation.
} 
Next we consider how the evaluation of truncated Chebyshev series is vectorized. First we review how the series is evaluated in the scalar code. Given an argument $x$ and a set of Chebyshev coefficients $c_{i}, i=1, \ldots, n$, the following algorithm due to Clenshaw evaluates

$$
f(x)=\frac{1}{2} c_{1} T_{0}(x)+\sum_{i=1}^{n-1} c_{i+1} T_{i}(x) .
$$

\section{Algorithm 1 : Clenshaw Recurrence - Scalar Version}

0. Initialize

$$
\begin{aligned}
& \beta \leftarrow 0 \\
& \gamma \leftarrow 0
\end{aligned}
$$

1. Recurrence for series

$$
\text { for } \begin{aligned}
i=n \text { step }-1 \text { to } 1 \text { do: } \\
\alpha \leftarrow \gamma \\
\gamma \leftarrow \beta \\
\beta \leftarrow 2 x \gamma-\alpha+c_{i}
\end{aligned}
$$

2. Last term; result is in $f$

$$
f \leftarrow(\beta-\alpha) / 2
$$

When we have a vector of arguments, $x_{j}, j=1, \ldots, m$, it is clear what should be done: $x, \alpha, \beta, \gamma$, and $f$ become vectors and all assignments are loops that run for $j=1, \ldots, m$. When this is done, however, the recurrence loop has two unnecessary vector copies. These can be eliminated when the loop on $i$ is unrolled to a level of three. This is illustrated in the following vector version of the above algorithm; note that three temporary vectors of length $m$ are required in addition to the input vector $x$ and the output vector $f$.

\section{Algorithm 2 : Clenshaw Recurrence - Vector Version}

\section{Initialize}

$$
\begin{aligned}
& k \leftarrow n \bmod 3 \\
& \beta_{j} \leftarrow 0 \text { for } j=1, \ldots, m \\
& \gamma_{j} \leftarrow 0 \text { for } j=1, \ldots, m \\
& f_{j} \leftarrow 2 x_{j} \quad \text { for } j=1, \ldots, m
\end{aligned}
$$

1. Recurrence for series (unrolled)

$$
\text { for } \begin{aligned}
& i=n \text { step }-3 \text { to } 1+k \text { do: } \\
& \alpha_{j} \leftarrow f_{j} \beta_{j}-\gamma_{j}+c_{i} \quad \text { for } j=1, \ldots, m \\
& \gamma_{j} \leftarrow f_{j} \alpha_{j}-\beta_{j}+c_{i-1} \quad \text { for } j=1, \ldots, m \\
& \beta_{j} \leftarrow f_{j} \gamma_{j}-\alpha_{j}+c_{i-2} \quad \text { for } j=1, \ldots, m
\end{aligned}
$$

2. Last term; cleanup for $n$ not divisible by three 
Table 1: User-callable VFNLIB subprograms.

\begin{tabular}{|cc|l|c|}
\hline \multicolumn{2}{|c|}{ VFNLIB } & \multicolumn{1}{c|}{ Description } & \multicolumn{2}{c|}{ FNLIB } \\
Single Double & \multicolumn{1}{|c|}{ Dingle Double } \\
\hline VI0 & DVI0 & Evaluates $I_{0}$ for a vector of arguments. & BESI0 DBESI0 \\
VI1 & DVI1 & Evaluates $I_{1}$ for a vector of arguments. & BESI1 DBESI1 \\
VJ0 & DVJ0 & Evaluates $J_{0}$ for a vector of arguments. & BES J0 DBESJ0 \\
VJ1 & DVJ1 & Evaluates $J_{1}$ for a vector of arguments. & BES J1 DBESJ1 \\
VK0 & DVK0 & Evaluates $K_{0}$ for a vector of arguments. & BESK0 DBESK0 \\
VK1 & DVK1 & Evaluates $K_{1}$ for a vector of arguments. & BESK1 DBESK1 \\
VY0 & DVY0 & Evaluates $Y_{0}$ for a vector of arguments. & BESY0 DBESY0 \\
VY1 & DVY1 & Evaluates $Y_{1}$ for a vector of arguments. & BESY1 DBESY1 \\
\hline
\end{tabular}

if $(k=0)$ then

$f_{j} \leftarrow\left(\beta_{j}-\alpha_{j}\right) / 2 \quad$ for $j=1, \ldots, m$

elseif $(k=1)$ then

$\alpha_{j} \leftarrow f_{j} \beta_{j}-\gamma_{j}+c_{1} \quad$ for $j=1, \ldots, m$

$f_{j} \leftarrow\left(\alpha_{j}-\gamma_{j}\right) / 2$ for $j=1, \ldots, m$

elseif $(k=2)$ then

$\alpha_{j} \leftarrow f_{j} \beta_{j}-\gamma_{j}+c_{2} \quad$ for $j=1, \ldots, m$

$\gamma_{j} \leftarrow f_{j} \alpha_{j}-\beta_{j}+c_{1} \quad$ for $j=1, \ldots, m$

endif

$f_{j} \leftarrow\left(\gamma_{j}-\beta_{j}\right) / 2$ for $j=1, \ldots, m$

The loop on $i$ repeatedly utilizes four vectors which can remain in vector registers for the entire computation. Unrolling the loops in this way yields a $20 \%$ to $25 \%$ improvement in the overall performance of our software on the Cray.

\section{The Vector Codes}

Using techniques described in the section above, we have produced portable vectorized routines for evaluating the following Bessel functions of real argument and orders zero and one: $I_{0}, I_{1}, J_{0}, J_{1}, K_{0}, K_{1}, Y_{0}$, and $Y_{1}$ [1]. Since our codes employ the basic algorithms of. FNLIB retooled for a vector environment we have called our collection of codes VFNLIB. Although we have produced new user-callable versions of only a small portion of the original FNLIB, the techniques which we have used can be readily applied to many of the remaining FNLIB routines.

VFNLIB includes the 16 user-callable Fortran subprograms which are listed in Table 1. Each has an identical calling sequence.

CALL name (M, X, F, WORK, IWORK, INFO) 
$\mathrm{X}$ is an array containing the $\mathrm{M}$ arguments for which the function is to be evaluated. The results are returned in the corresponding positions of the array F. WORK and IWORK are workspace arrays. IWORK is of length $\mathrm{M}$, while WORK is of length 7M. INFO is a return code. Both single precision and double precision versions of each function are provided.

We have elected to provide an error return code parameter rather than issue all error messages through an error handler as is done by FNLIB. Error conditions are indicated by a nonzero return code INFO. Fatal errors are indicated by INFO $>0$, while warnings have INFO < 0 . If any argument leads to a fatal error then no results are returned. Fatal error conditions are: $M \leq 0$, function undefined for an argument, a result overflows, an argument so large that accurate argument reduction is not possible. In the last three cases the index of the first offending argument is returned in IWORK(1). A warning is issued when any result underflows; the corresponding function value is set to zero in this case.

We have used a separate procedure to handle errors detected in lower-level routines. An example is that an $n$-term Chebyshev series is to be evaluated for $n<1$. Such errors are not caused by improper user input and should never occur during the normal use of these routines. (The exception is when the array wORK is too short and the code overwrites other data.) The only reasonable thing to do in these cases is to issue a fatal error message and halt the program. This is done by calling an very simple error-handling routine WFERR.

A list of all subsidiary routines included in VFNLIB is given in Table 2. All codes are written in standard Fortran 77. A number of common operations such as vector index compression, vector gather and vector scatter are used in VFNLIB. These operations are the most likely to lead to compiler vectorization failures. Although they each vectorize on both the Convex and the Cray Y-MP, they did not vectorize on the Cyber 205, for example. As a result, we have encapsulated these operations into low-level utilities which, if necessary, can be replaced by processor-dependent utilities.

\section{Evaluation}

\subsection{Portability and Accuracy}

Since VFNLIB represents a vectorization of a subset of FNLIB, our first goal is to assure that VFNLIB routines return the same results as the corresponding FNLIB routines. A test program that is distributed with the package compares each function with its FNLIB counterpart for approximately 23,000 arguments, verifying that the two differ by no more than five times the machine epsilon. (Relative difference is used for function values greater than one, absolute difference for those less than one.) This code was run on three separate computer systems: Sun SPARCstation 1+ (Sun OS 4.0.3) using Sun Fortran 1.3.1 (options -fast -O3), Convex C-120 (Convex Unix 4.2 release 9.0) using Convex fc version V6.1 (option -O2), Cray Y-MP2/216 (UNICOS 6.0) using cf77. In each case available options were used to check for conformance to ANSI X3.9-1978 FORTRAN.

Some additional testing was done to verify that both sets of routines were indeed producing correct function values. Ideally, function routines return results with relative error near the machine precision. This is rarely the case in practice. The accuracy of the VFNLIB 
Table 2: Internal VFNLIB subprograms.

\begin{tabular}{|c|c|c|c|c|}
\hline \multicolumn{2}{|c|}{ VFNLIB } & Description & \multicolumn{2}{|c|}{$\begin{array}{c}\text { FNLIB } \\
\text { Single Double }\end{array}$} \\
\hline WIO & DWIO & Evaluates $I_{0}$ for a vector of arguments. & & \\
\hline WI1 & DWI1 & Evaluates $I_{1}$ for a vector of arguments. & & \\
\hline WJO & DWJO & Evaluates $\mathrm{J}_{0}$ for a vector of arguments. & & \\
\hline WJ 1 & DWJ 1 & Evaluates $J_{1}$ for a vector of arguments. & & \\
\hline WKO & DWKO & Evaluates $K_{0}$ for a vector of arguments. & & \\
\hline WK1 & DWK1 & Evaluates $K_{1}$ for a vector of arguments. & & \\
\hline WYO & DWYO & Evaluates $Y_{0}$ for a vector of arguments. & & \\
\hline WY1 & DWY1 & Evaluates $Y_{1}$ for a vector of arguments. & & \\
\hline WCS & DWCS & $\begin{array}{l}\text { Evaluates a given Chebyshev series for a } \\
\text { vector of arguments. }\end{array}$ & CSEVL & DCSEVL \\
\hline IWCS & IDWCS & $\begin{array}{l}\text { Determines number of terms necessary to } \\
\text { compute a given Chebyshev series to a } \\
\text { given accuracy. }\end{array}$ & INITS & INITDS \\
\hline WNGT & DWNGT & $\begin{array}{l}\text { Determines if elements of a vector are } \\
\text { greater than a given scalar. }\end{array}$ & & \\
\hline WNLE & DWNLE & $\begin{array}{l}\text { Determines if elements of a vector are less } \\
\text { than or equal to a given scalar. }\end{array}$ & & \\
\hline WGT & DWGT & $\begin{array}{l}\text { Index compression. Given a vector } x_{i} \text {, and } \\
\text { scalars } a \text { and } b \text {, this constructs an array of } \\
\text { indices } j \text { for which } x_{j}>b \text {. }\end{array}$ & & \\
\hline WLE & DWLE & $\begin{array}{l}\text { Index compression. Given a vector } x_{i} \text {, and } \\
\text { scalars } a \text { and } b \text {, this constructs an array of } \\
\text { indices } j \text { for which } x_{j} \leq b \text {. }\end{array}$ & & \\
\hline WGTLE & DWGTLE & $\begin{array}{l}\text { Index compression. Given a vector } x_{i} \text {, and } \\
\text { scalars } a \text { and } b \text {, this constructs an array of } \\
\text { indices } j \text { for which } a<x_{j} \leq b \text {. }\end{array}$ & & \\
\hline WGTHR & DWGTHR & Vector gather. & & \\
\hline WSCTR & DWSCTR & Vector scatter. & & \\
\hline WFERR & & Processes a fatal error message. & SETERU & \\
\hline I1MACH & I 1MACH & $\begin{array}{l}\text { Returns integer valued machine dependent } \\
\text { constants. From PORT [8]. }\end{array}$ & I1MACH & I1MACH \\
\hline R1MACH & D1MACH & $\begin{array}{l}\text { Returns real valued machine dependent } \\
\text { constants. From PORT [8]. }\end{array}$ & R1MACH & D1MACH \\
\hline
\end{tabular}


routines was assessed by evaluating each function for four to five thousand arguments on the Cray Y-MP with both FNLIB and VFNLIB and then comparing the results with those computed in extended precision using Mathematica [11]. In most cases the results returned by FNLIB and VFNLIB have relative error less than about 10 times the machine epsilon $\left(1.4 \times 10^{-14}\right.$ in single precision, $1.0 \times 10^{-28}$ in double precision $)$. Two notable exceptions to this behavior occur for the functions $J_{0}, J_{1}, Y_{0}$, and $Y_{1}$, which we describe below.

Cancellation at function zeros. These functions are oscillatory, and cancellation errors in the evaluation of the Chebyshev series lead to a loss of relative accuracy in the neighborhood of their zeros (except for $J_{1}(0)=0$ ). In these cases the absolute error remains less than about 10 times the machine epsilon.

Argument reduction for sine and cosine. For large arguments, these functions are computed as $A(x) g(\theta(x))$, where $A$ and $\theta$ are given by Chebyshev series, and $g$ is a sine or cosine. Since $\theta(x)$ is of the same size as $x$, the sine or cosine of a large argument must be computed when $x$ is large. Argument reduction within the sine and cosine procedures can lead to loss of precision for large $x$.

\subsection{Efficiency}

The chief motivation for using VFNLIB is the prospect of improved computation rates on vector processors. To assess the advantage of using VFNLIB, we compare the use of VFNLIB routines ("vector codes") with repeated calls of corresponding FNLIB routines ("scalar codes"). We compare the codes on the three systems described in Section 4: Sun SPARCstation 1+, Convex C-120, and Cray Y-MP2. The first is a scalar workstation, the second a vector minisupercomputer, and the third an 2-processor vector supercomputer (although we only use one processor in these tests). We include the Sun to demonstrate the portability of the software, as well as to show that use of VFNLIB need not degrade performance on on scalar processors. All tests were performed in single precision (the Sun and Convex are 32-bit machines while the Cray is a 64-bit machine).

The computation in the innermost loop of the vectorized codes is the evaluation of Chebyshev series using the algorithm of Section 3. In Table 3 we list the computation rate in megaflops for evaluating a single Chebyshev series for 2000 arguments using both scalar and vector codes. The subprograms CSEVL and WCS were used for this purpose. The theoretical peak rate for the Convex and Cray are 40 and 333 megaflops, respectively, while the corresponding Linpack benchmark runs at 6.5 and 90 megaflops [5]. In light of these figures, the observed rate for vectorized Chebyshev series evaluation on the Convex and Cray, 15 and 182 , respectively, are seen to be quite reasonable.

Unfortunately, as we have seen, there is more to the computation of Bessel functions than the evaluation of Chebyshev series. Overhead must be paid for the gathering and scattering of vector elements, as well as for various error checks. It is easy to assess the effect of this overhead on the Cray Y-MP using its hardware performance monitor. Table 4 lists the measured asymptotic computation rate in megaflops for each user-callable function. To obtain this data, we evaluated each function for 19 different sets of 2000 arguments, 
Boisvert and Saunders

Table 3: Asymptotic Performance of Chebyshev Series Evaluation (Megaflops)

\begin{tabular}{|l|r|rr|}
\hline Machine & Terms & Scalar & Vector \\
\hline Sun SPARC 1+ & 7 & 1.8 & 2.9 \\
Convex C-120 & 7 & 1.2 & 15.0 \\
Cray Y-MP2 & 20 & 12.7 & 182.1 \\
\hline
\end{tabular}

Table 4: Asymptotic Performance of Bessel Functions on Cray Y-MP (Megaflops)

\begin{tabular}{|l|rrrrrrrr|}
\hline Function & $\mathrm{I}_{0}$ & $\mathrm{I}_{1}$ & $\mathrm{~J}_{0}$ & $\mathrm{~J}_{1}$ & $\mathrm{~K}_{0}$ & $\mathrm{~K}_{1}$ & $\mathrm{Y}_{0}$ & $\mathrm{Y}_{1}$ \\
\hline Scalar & 9.0 & $S . \bar{\tau}$ & 10.6 & 10.4 & $7 . S$ & $S .0$ & 9.1 & 9.0 \\
Vector & $14 S .7$ & 142.4 & 164.5 & 160.9 & 162.8 & 159.3 & 172.0 & 170.0 \\
\hline
\end{tabular}

each with a different argument distribution. Comparing Table 3 and Table 4 we see that computation rates for full Bessel function evaluation decline only 7 to 22 percent over raw Chebyshev series evaluation.

Another measure of performance is speedup, the ratio of scalar to vector times for multiple function evaluation. Actual speedups depend upon the distribution of arguments. We compare the scalar and vector codes for vectors of length 2000 distributed in 19 different ways among three different argument ranges. The test includes cases where all arguments are in a single range as well as cases where only one per cent of the arguments are in a given range. Table 5 lists minimum, average, and maximum speedups observed with the single precision codes on each computer. The speedups for the Convex are in the range of 4.4 to 9.6 while the speedups on the Cray are in the range 13.9 to 23.0. (The entry labelled "Convex C-120 with VECLIB" will be explained shortly.) It is also interesting to note that the vectorized codes run faster than the scalar codes on the Sun in most cases.

The average speedups for the Cray Y-MP given in Table 5 are somewhat better than one might predict from the computation rates given in Table 3 . In contrast, the speedups for the Convex are not as large as one might predict. To investigate this further we generated an execution profile of the subroutine VI0 evaluating $I_{0}$ for a vector of length 2000 with $x_{i}=1$ for all $i$. The first two columns of Table 6 show the percent time spent by the Cray and the Convex on four different activities. The innermost loop of the computation is Chebyshev series evaluation, which is where more than half the computation occurs on the Cray. However, on the Convex, more than half the time is spent doing index compression; although the compiler does "vectorize" the index compression loops, the resulting code does not perform very well.

In VFNLIB operations such as index compression and gather/scatter have been isolated in low-level utility routines so that they can be easily replaced on systems whose Fortran compilers cannot adequately vectorize them. On the Convex the index compression and gather/scatter loops may be replaced with calls to VECLIB library which has been optimized 
Table 5: Asymptotic Speedups (Scalar time / vector time)

\begin{tabular}{|l|l|rrrrrrrr|}
\hline Machine & & $\mathrm{I}_{0}$ & $\mathrm{I}_{1}$ & $\mathrm{~J}_{0}$ & $\mathrm{~J}_{1}$ & $\mathrm{~K}_{0}$ & $\mathrm{~K}_{1}$ & $\mathrm{Y}_{0}$ & $\mathrm{Y}_{1}$ \\
\hline Sun SPARC 1+ & Min & 1.0 & 1.0 & 1.1 & 1.1 & 1.0 & 1.0 & 1.2 & 1.2 \\
& Ave & 1.1 & 1.1 & 1.2 & 1.2 & 1.2 & 1.2 & 1.3 & 1.3 \\
& Max & 1.2 & 1.2 & 1.4 & 1.2 & 1.3 & 1.4 & 1.6 & 1.5 \\
\hline Convex C-120 & Min & 4.4 & 5.2 & 5.5 & 4.6 & 7.6 & 7.3 & 7.9 & 7.9 \\
& Ave & 5.8 & 5.9 & 6.4 & 5.6 & 8.0 & 8.0 & 8.4 & 8.5 \\
& Max & 6.5 & 6.2 & 6.8 & 6.0 & 8.3 & 9.1 & 9.3 & 9.6 \\
\hline Convex C-120 & Min & 6.2 & 7.5 & 6.6 & 5.8 & 8.2 & 9.1 & 8.6 & 8.0 \\
with VECLIB & Ave & 7.9 & 7.9 & 7.3 & 6.6 & 9.8 & 9.6 & 9.2 & 8.6 \\
& Max & 8.8 & 8.2 & 7.6 & 7.0 & 10.9 & 10.4 & 10.3 & 9.7 \\
\hline Cray Y-MP2 & Min & 16.2 & 15.3 & 14.5 & 13.9 & 20.8 & 20.9 & 14.8 & 14.9 \\
& Ave & 17.8 & 16.8 & 16.0 & 15.3 & 21.5 & 21.6 & 19.6 & 19.8 \\
& Max & 20.7 & 19.9 & 21.5 & 20.4 & 22.6 & 23.1 & 23.4 & 23.0 \\
\hline
\end{tabular}

Table 6: Percent Time Spent by VI0 on Various Activities

\begin{tabular}{|l|rrc|}
\hline Activity & Cray & Convex & Convex with VECLIB \\
\hline Chebyshev series evaluation & 57.7 & 22.8 & 34.8 \\
Pre- and post- processing & 12.1 & 10.2 & 15.8 \\
Index compression & 20.0 & 50.2 & 20.2 \\
Gather/scatter & 5.2 & 9.7 & 17.9 \\
\hline
\end{tabular}


Table 7: Short Vector Speedups (Scalar time / vector time)

\begin{tabular}{|l|r|rrrrrrrr|}
\hline Machine & length & $\mathrm{I}_{0}$ & $\mathrm{I}_{1}$ & $\mathrm{~J}_{0}$ & $\mathrm{~J}_{1}$ & $\mathrm{~K}_{0}$ & $\mathrm{~K}_{1}$ & $\mathrm{Y}_{0}$ & $\mathrm{Y}_{1}$ \\
\hline Sun SPARC 1+ & 2 & 0.5 & 0.5 & 0.5 & 0.5 & 0.6 & 0.6 & 0.6 & 0.6 \\
& 20 & 1.2 & 1.0 & 1.0 & 1.2 & 1.2 & 1.2 & 1.1 & 1.2 \\
\hline Convex C-120 & 2 & 0.2 & 0.2 & 0.2 & 0.2 & 0.3 & 0.3 & 0.2 & 0.2 \\
& 20 & 1.8 & 1.8 & 1.8 & 1.6 & 2.2 & 2.3 & 2.0 & 2.2 \\
\hline Cray Y-MP2 & 2 & 0.4 & 0.4 & 0.4 & 0.4 & 0.5 & 0.5 & 0.4 & 0.5 \\
& 20 & 3.6 & 3.5 & 3.5 & 3.4 & 4.6 & 4.5 & 4.0 & 4.0 \\
\hline
\end{tabular}

Table S: Short Vector Break-even Point (Vector time = scalar time)

\begin{tabular}{|l|rrrrrrrr|}
\hline Machine & $\mathrm{I}_{0}$ & $\mathrm{I}_{1}$ & $\mathrm{~J}_{0}$ & $\mathrm{~J}_{1}$ & $\mathrm{~K}_{0}$ & $\mathrm{~K}_{1}$ & $\mathrm{Y}_{0}$ & $\mathrm{Y}_{1}$ \\
\hline Sun SPARC 1+ & 12 & 12 & 14 & 10 & 8 & 8 & 7 & 7 \\
Convex C-120 & 10 & 10 & 10 & 11 & 8 & 7 & 9 & 8 \\
Cray Y-MP2 & 5 & 5 & 5 & 5 & 4 & 4 & 5 & 5 \\
\hline
\end{tabular}

for use on the Convex [4]. When this is done the entries in the third column of Table 6 are obtained. This is is a more reasonable distribution, although the gather/scatter operations now occupy a larger percentage of time than on the Cray. Using the modified version of VFNLIB's utilities on the Convex leads to the the improved scalar/vector speedups labelled "Convex C-120 with VECLIB" in Table 5.

The tests that we have performed thus far assess the performance of the codes for very long vectors. It is also important to assess the penalty for using the vectorized codes when vector lengths are short. Table 7 lists sample speedups for each function on the three machines for vectors of length 2 and 20. Arguments for these tests are distributed over two argument subranges so that internal vector lengths are actually half that listed in the table. As one would expect, the vector codes run slower in each case when very few function values are requested. For vector length 2 the vector code runs twice as long on the Sun and the Cray, and five times as long on the Convex. Table 8 lists the vector length for which the speedup is 1, i.e., the break-even point after which the vector codes are faster. The breakeven point is in the range 4-5 for the Cray, 7-11 for the Convex, and 7-14 for the Sun.

\section{Conclusion}

We have described a suite of portable Fortran subprograms, VFNLIB, for computing the Bessel functions of real argument and integer order. The codes are a modification of routines from Fullerton's well-known FNLIB package. The new routines allow users to specify an array of arguments at which to evaluate the functions, and the algorithms have been changed to allow effective vectorization when this is the case. Speedups of from 13 to 22 
over use of the original FNLIB have been observed for the VFNLIB codes on the Cray Y-MP. Modest speedups were also observed on the Sun SPARCstation 1+, a scalar workstation. The vectorization techniques employed in VFNLIB extend to many other FNLIB routines.

\section{Disclaimer}

Identification of commercial products in this paper does not imply recommendation or endorsement by NIST.

\section{References}

[1] M. Abramowitz and I. A. Stegun, editors. Handbook of Mathematical Functions with Formulas, Graphs, and Mathematical Tables. U. S. Government Printing Office, Washington, D.C., 1964.

[2] B. L. Buzbee. The SLATEC common mathematical library. In W. R. Cowell, editor, Sources and Development of Mathematical Software, pages 302-318. Prentice-Hall, Englewood Cliffs, N.J, 1984.

[3] C. W. Clenshaw. A note on the summation of Chebyshev series. M. T. A. C., 9:118-120, 1955.

[4] Convex Computer Corporation, Richardson, TX. CONVEX VECLIB User's Guide, third edition, October 1988.

[5] J. J. Dongarra. Performance of various computers using standard linear equations software. Computer Science Department Report CS-89-85, University of Tennessee, August 1990.

[6] J. J. Dongarra and E. Grosse. Distribution of mathematical software via electonic mail. Comm. ACM, 30:403-407, 1987.

[7] L. Fox and I. B. Parker. Chebyshev Polynomials in Numerical Analysis. Oxford University Press, London, 1979.

[8] P. A. Fox, A. D. Hall, and N. L. Schryer. Algorithm 528: Framework for a portable library. ACM Trans. Math. Softw., 4:177-188, 1978.

[9] L. W. Fullerton. Portable special function routines. In W. Cowell, editor, Portability of Numerical Software, volume 57 of Lecture Notes in Computer Science, pages 452-483. Springer-Verlag, New York, 1976.

[10] IMSL Inc., 2500 CityWest Blvd., Houston, TX 77042-3020. SFUN/LIBRARY: FORTRAN Subroutines for Evaluating Special Functions, April 1987. Version 2.0. 
[11] S. Wolfram. Mathematica, A System for Doing Mathematics by Computer. AddisonWesley, Redwood City, CA, $198 \mathrm{~S}$. 
NIST-114A

(REV. 3-90)
U.S. DEPARTMENT OF COMMERCE

NATIONAL INSTITUTE OF STANDARDS AND TECHNOLOGY

\section{BIBLIOGRAPHIC DATA SHEET}

1. PUEUCATION OR REPORT MUMBER NISTIR 4615

2. PERFORMING ORGAMIZATION REPORT NUMBER

3. PUELCATION DAT

JUNE 1991

\section{TITLE AND SUBTITLE}

Portable Vectorized Software for

Bessel Function Evaluation

5. AUTHOR(\$)

Ronald F. Boisvert and Bonita V. Saunders

6. PERFORMING ORGAMIZATIOH (IF JOINT ON OTHER THAN MIST, SEE INSTRUCTIONS)

U.S. DEPARTMENT OF COMLERCE

MATIONAL INSTITUTE OF STANDARDS AND TECHMOLOCY

GATHERSBURG, MD 20090

7. CONTRACT/GRANT MUMBER

2. TYPE OF REPORT AND PERIOD COVERED

9. SPONSORING ORQANIZATION MAME AND COMPLETE ADORESS (STREET, CTT, STATE, ZP)

11. ABSTRACT (A 200-WORD ON LESS FACTUML SUMMAAY OF MOST SIGMIFICANT IMFORMATIOM. IF DOCUMENT IMCLUDES A SIGNIFICANT BIBUOGRAPHY OR UTERATURE SURVEY, MENTON IT HERE)

A sulte of computer programs for the evaluation of Bessel functions and modified Bessel functions of orders zero and one for a vector of real arguments is described. Distinguishing characteristics of these programs are that (a) they are portable across a wide range of machines, and (b) they are vectorized in the case when multiple function evaluations are to be performed. The performance of the new programs are compared with software from the FNLIB collection of Fullerton on which the new software is based.

12. KEY WORDS (6 TO 12 ETTRIES; NLPLETICAL ORDER; CAPTTALEE ONLY PROPER MMES; AND SEPARATE KEY WORDS BY SEMICOLONS) Bessel function; hyperbolic Bessel function; mathematical software; modified Bessel function; order zero and one; portable software; special function; vectorized software

13. AVALABLTY

\section{$\mathrm{X}$ UNUMTTED}

FOR OFFICLLL DISTRIAUTIOH. DO MOT RELASE TO MATIONAL TECHMICAL INFORMATION SERVICE (NTTS).

ORDER FROM SUPEAHTENDENT OF DOCUMENTS, U.S. GOVERMMENT PRINTING OFFICE, WASHINOTON, DC 20402

14. NUMBER OF PAINTED PAOES 



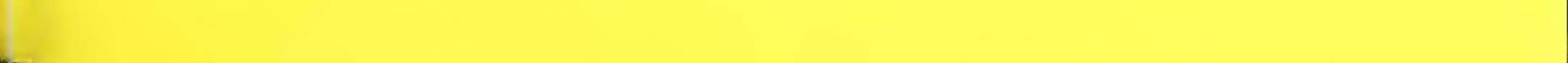


